\title{
One-Pot Synthesis of $4 \mathrm{H}$-Chromene and Dihydropyrano[3,2-c]chromene Derivatives in Hydroalcoholic Media
}

\author{
Ramin Ghorbani-Vaghei,* Zahra Toghraei-Semiromi and Rahman Karimi-Nami
}

\author{
Department of Organic Chemistry, Faculty of Chemistry, Bu-Ali Sina University, 65174 Hamedan, Iran
}

\begin{abstract}
4H-Cromenos e diidropirano[3,2-c]cromenos são obtidos em rendimentos bons a excelentes através de um procedimento simples, brando e eficiente usando poli $\left(N, N^{\prime}\right.$-dibromo- $N$-etil-benzeno1,3-dissulfonamida) [PBBS] e $N, N, N^{\prime}, N^{\prime}$-tetrabromobenzeno-1,3-dissulfonamida [TBBDA] como catalisadores.
\end{abstract}

$4 H$-Chromenes and dihydropyrano[3,2-c]chromenes are obtained in good to excellent yields by a simple, mild and efficient procedure using poly $\left(N, N^{\prime}\right.$-dibromo- $N$-ethyl-benzene1,3-disulfonamide) [PBBS] and $N, N, N^{\prime}, N$ '-tetrabromobenzene-1,3-disulfonamide [TBBDA ] as catalysts.

Keywords: $4 H$-chromenes, dihydropyrano[3,2-c]chromenes, TBBDA, PBBS

\section{Introduction}

The development of multi-component reactions (MCRs) has attracted much attention from the vantage point of combinatorial and medicinal chemistry. ${ }^{1}$ Many important heterocycle syntheses are multi-component reactions. Recently, the synthesis of $4 H$-chromenes and dihydropyrano $[3,2-c]$ chromenes derivatives have attracted great interest to their biological and pharmacological activities. The $4 H$-chromene derivatives show various pharmacological properties such as spasmolytic, diuretic, anticoagulant, anticancer, and antianaphylactic activities. ${ }^{2}$ Substituted $4 H$-chromenes are particularly versatile compounds that bind Bcl-2 protein (B-cell lymphoma 2) and induce apoptosis in tumor cells. Specifically, Bcl-2 can contribute to neoplastic cell expansion by preventing normal cell turnover caused by physiological cell death mechanisms. High levels of the Bcl-2 gene expressions are found in a wide variety of human cancers and can lead to tumor cell resistance to conventional chemotherapy and radiotherapy. Thus, $\mathrm{Bcl}-2$ protein binding compounds provide a promising lead for the development of potential anticancer agents and direct methods for their synthesis are highly desirable. ${ }^{3-5}$ Dihydropyrano $[3,2-c]$ chromenes are a class of important heterocycles that have been used as cognitive enhancers, for the treatment of neurodegenerative

*e-mail: rgvaghei@yahoo.com diseases, including Alzheimers disease, amyotrophic lateral sclerosis, Parkinson's disease, Huntington's disease, AIDS associated dementia and Down's syndrome as well as for the treatment of schizophrenia and myoclonus. ${ }^{6}$ In addition, aminochromene derivatives exhibit a wide spectrum of biological activities including antihypertensive and anti-ischemic behavior. ${ }^{7-9}$ Also, a number of 2-amino- $4 \mathrm{H}$-pyrans are useful as photoactive materials. ${ }^{10}$

$4 H$-Chromenes have been prepared from salicylaldehydes and cyanoacetates in heterogeneous liquid phase catalysis using $\mathrm{Al}_{2} \mathrm{O}_{3}{ }^{4,11}$ and molecular sieves. ${ }^{5}$ They are also synthesized in the presence of $\mathrm{Zr}\left(\mathrm{KPO}_{4}\right)_{2}{ }^{12}$ and Amberlyst A2 $1{ }^{\oplus} \cdot{ }^{13}$ Despite their importance from pharmacological, industrial and synthetic point of views, comparatively few methods for accessing pyrano[3,2-c]chromene derivatives have been reported. ${ }^{14-16}$ 2-Amino-4-aryl-5-oxo-4H, $5 H$-pyrano-[3,2-c]chromene-3-carbonitriles have already been prepared in the presence of organic bases like piperidine or pyridine in an organic solvent, i.e., ethanol and pyridine. ${ }^{14}$ They are also obtained in the presence of diammonium hydrogen phosphate, ${ }^{15} \mathrm{H}_{6} \mathrm{P}_{2} \mathrm{~W}_{18} \mathrm{O}_{62} \cdot 18 \mathrm{H}_{2} \mathrm{O},{ }^{16} \mathrm{DBU}^{17}$ and $\mathrm{K}_{2} \mathrm{CO}_{3}$ under microwave irradiation. ${ }^{18}$

However, some of these protocols require long reaction times, multi-step reactions, complex synthetic pathways and afford products with only modest yields. Therefore, the introduction of milder, faster and more ecofriendly methods, accompanied with higher yields is needed. 


\section{Results and Discussion}

In continuation of our interest in the application of $N, N, N^{\prime}, N^{\prime}$-tetrabromo benzene-1,3-disulfonamide [TBBDA] and poly $\left(N, N^{\prime}\right.$-dibromo- $N$-ethyl-benzene-

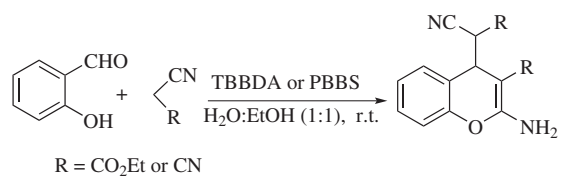

Scheme 1. 1,3-disulfonamide) $[\mathrm{PBBS}]^{19-22}$ in organic synthesis, we report here a convenient method for the preparation of $4 H$-chromenes from salicylaldehydes and malononitrile or ethyl cyanoacetate in aqueous ethanol $\left(\mathrm{H}_{2} \mathrm{O}: \mathrm{EtOH},(1: 1)\right)$ at room temperature (Scheme 1 and Table 1).

Also the synthesis of dihydropyrano[3,2-c]chromenes was achieved by the three-component condensation of an aromatic aldehyde, malononitrile and 4-hydroxycoumarin in the presence of the catalysts. The reaction was carried out in aqueous ethanol at reflux using TBBDA and PBBS as catalysts to give products in good to high yields (Scheme 2 and Table 2).

Table 1. Synthesis of various $4 H$-chromenes using TBBDA and PBBS at room temperature

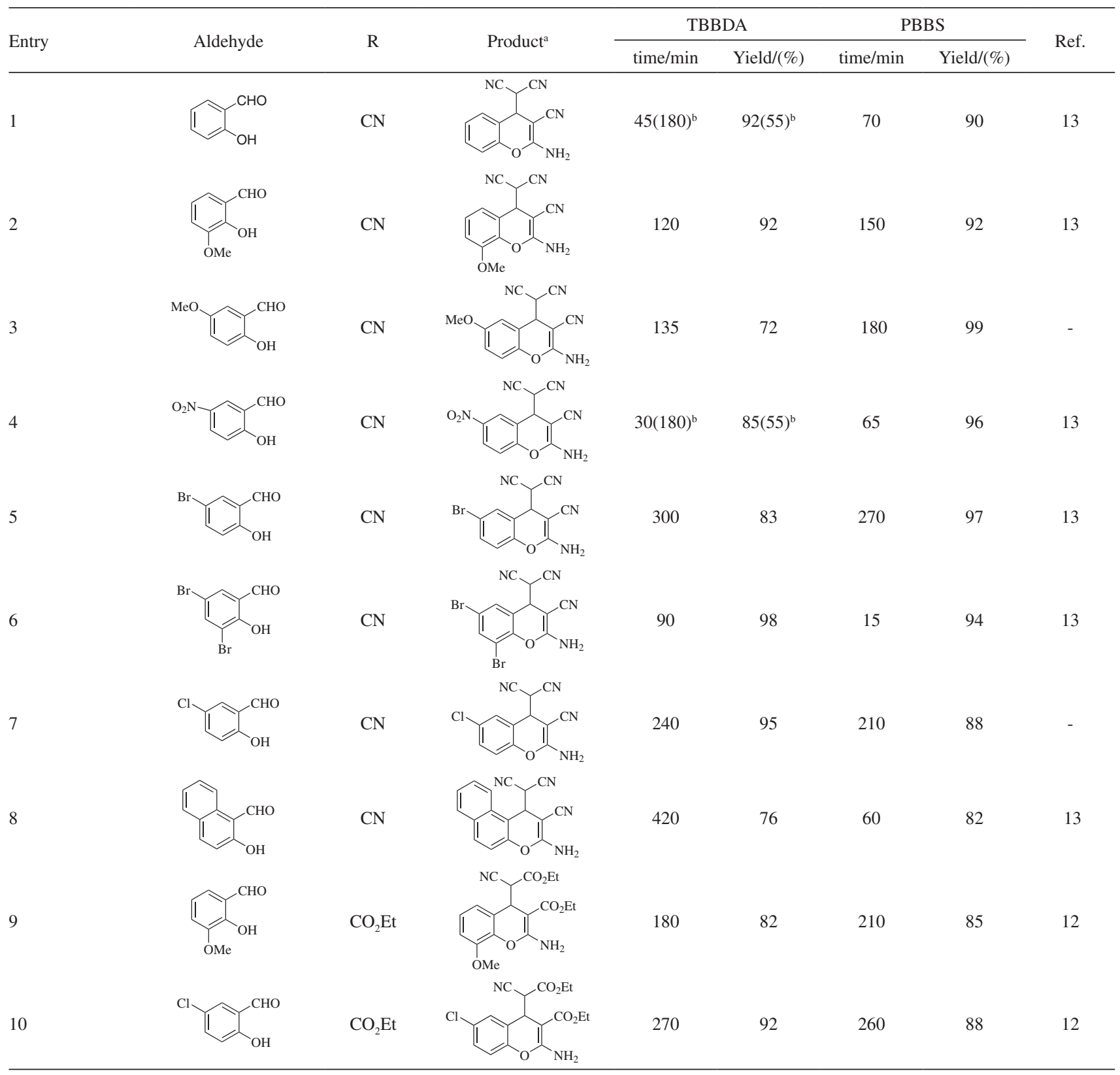

aProducts were characterized from their physical properties, by comparison with authentic samples, and by spectroscopic methods. ${ }^{\mathrm{b}}$ Without using the catalysts. 


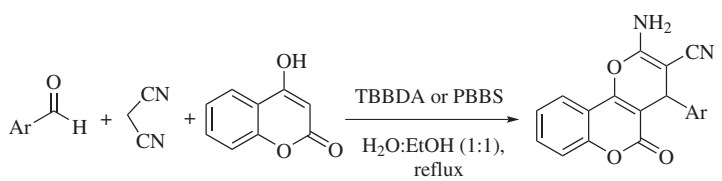

Scheme 2.

Table 2. Synthesis of various dihydropyrano[3,2-c]chromenes using TBBDA and PBBS under refluxing $\mathrm{H}_{2} \mathrm{O}: \mathrm{EtOH}(1: 1)$

\begin{tabular}{|c|c|c|c|c|c|c|c|}
\hline \multirow{2}{*}{ Entry } & \multirow{2}{*}{$\mathrm{ArCHO}$} & \multirow{2}{*}{ Product $^{\mathrm{a}}$} & \multicolumn{2}{|c|}{ TBBDA } & \multicolumn{2}{|c|}{ PBBS } & \multirow{2}{*}{ Ref } \\
\hline & & & time/min & Yield/(\%) & time/min & Yield/(\%) & \\
\hline 1 & $\mathrm{PhCHO}$ & & 150 & 88 & 120 & 75 & 15 \\
\hline 2 & 4- $\mathrm{Cl}-\mathrm{C}_{6} \mathrm{H}_{4} \mathrm{CHO}$ & & 195 & 89 & 180 & 90 & 15 \\
\hline 3 & $4-\mathrm{OMe}-\mathrm{C}_{6} \mathrm{H}_{4} \mathrm{CHO}$ & & 75 & 81 & 150 & 73 & 15 \\
\hline 4 & 4-Me- $\mathrm{C}_{6} \mathrm{H}_{4} \mathrm{CHO}$ & & 50 & 72 & 200 & 79 & 17 \\
\hline 5 & $4-\mathrm{NO}_{2}-\mathrm{C}_{6} \mathrm{H}_{4} \mathrm{CHO}$ & & $170(240)^{\mathrm{b}}$ & $91(40)^{\mathrm{b}}$ & 150 & 90 & 15 \\
\hline 6 & $3-\mathrm{NO}_{2}-\mathrm{C}_{6} \mathrm{H}_{4} \mathrm{CHO}$ & & 180 & 97 & 240 & 83 & 15 \\
\hline 7 & 4- $\mathrm{Br}-\mathrm{C}_{6} \mathrm{H}_{4} \mathrm{CHO}$ & & 120 & 92 & 90 & 82 & 15 \\
\hline 8 & 2,4- $\mathrm{Cl}_{2}-\mathrm{C}_{6} \mathrm{H}_{4} \mathrm{CHO}$ & & 75 & 76 & 25 & 80 & 15 \\
\hline 9 & $2,3-\mathrm{Cl}_{2}-\mathrm{C}_{6} \mathrm{H}_{4} \mathrm{CHO}$ & & 60 & 88 & 20 & 82 & 15 \\
\hline 10 & 2,6- $\mathrm{Cl}_{2}-\mathrm{C}_{6} \mathrm{H}_{4} \mathrm{CHO}$ & & 90 & 90 & 30 & 94 & 5 \\
\hline 11 & $3,4,5-(\mathrm{OMe})_{3}-\mathrm{C}_{6} \mathrm{H}_{4} \mathrm{CHO}$ & & 300 & 89 & 330 & 92 & - \\
\hline
\end{tabular}


The advantages of PBBS and TBBDA are: (i) ease of preparation; (ii) reagent stability under atmospheric conditions for two months; (iii) possibility of re-use.

In conclusion, we have developed an efficient procedure for the synthesis of $4 \mathrm{H}$-chromenes and dihydropyrano[3,2-c]chromenes derivatives in aqueous media using [TBBDA] and [PBBS]. This method offers several advantages such as inexpensive catalysts, easy synthetic procedure, high yields, simple work-up procedure and easy product isolation.

\section{Experimental}

All commercially available chemicals were obtained from Merck and Fluka, and used without further purifications unless otherwise stated. ${ }^{1} \mathrm{H}$ NMR and ${ }^{13} \mathrm{C}$ NMR spectra were recorded on a Jeol $90 \mathrm{MHz}$ and a Bruker $300 \mathrm{MHz}$ FT NMR spectrometers using TMS as internal standard and chemical shifts in $\delta(\mathrm{ppm})$. Infrared (IR) spectra were acquired on a Perkin Elmer GX FT-IR spectrometer. All yields refer to isolated products.

\section{General procedure for the preparation of $4 \mathrm{H}$-chromenes}

A mixture of salicylaldehyde (5 mmol), malononitrile $(1.2 \mathrm{mmol})$ and TBBDA $(0.18 \mathrm{mmol})$ or PBBS $(0.1 \mathrm{~g})$ in $\mathrm{H}_{2} \mathrm{O}(5 \mathrm{~mL})$ and $\mathrm{EtOH}(5 \mathrm{~mL})$ was stirred at room temperature for the appropriate time. After completion of the reaction, which was monitored by TLC, the solid product was collected by filtration, washed with water and aqueous ethanol and purified by recrystallization from ethanol.

General procedure for the preparation of 2-amino-5-oxodihydropyrano[3,2-c]chromenes

A mixture of aldehyde $(10 \mathrm{mmol})$, malononitrile (1.2 mmol), 4-hydroxycoumarin (10 mmol) and TBBDA $(0.18 \mathrm{mmol})$ or PBBS $(0.1 \mathrm{~g})$ in $\mathrm{H}_{2} \mathrm{O}(5 \mathrm{~mL})$ and $\mathrm{EtOH}$ $(5 \mathrm{~mL})$ was stirred under reflux for the appropriate time. After completion of the reaction, which was monitored by TLC, the mixture was monitored by TLC, the mixture was cooled to room temperature. The solid product was collected by filtration, washed with water and aqueous ethanol and purified by recrystallization from ethanol.

\section{Recycling of the catalysts}

The catalysts were recovered by evaporation of the solvent and washing of the solid with dichloromethane.

\section{Supplementary Information}

Supplementary characterization data and ${ }^{1} \mathrm{H}$ NMR spectra are available, free of charge at http://jbcs.sbq.org.br as a PDF file.

\section{Acknowledgments}

We are thankful to Bu-Ali Sina University, Center of Excellence in Development of Chemical Methods (CEDCM) for financial support.

\section{References}

1. Domling, A.; Ugi, I.; Angew. Chem., Int. Ed. 2000, 39, 3168; Brase, S.; Gil, C.; Knepper, K.; Bioorg. Med. Chem. 2002, 10, 2415.

2. Foye, W. O.; Principi di Chemico Farmaceutica, Piccin: Padova, Italy, 1991; p. 416; Andreani, L. L.; Lapi, E.; Bull. Chim. Farm. 1960, 99, 583; Zhang, Y. L.; Chen, B. Z.; Zheng, K. Q.; Xu, M. L.; Lei, X. H.; Yао Хие Хие Ваo, 1982, 17, 17; Chem. Abstr. 1982, 69, 135383e; Bonsignore, L.; Loy, G.; Secci. D.; Calignano, A.; Eur. J. Med. Chem. 1993, 28, 517.

3. Yu, N.; Aramini, J. M.; Germann, M. W.; Huang, Z.; Tetrahedron Lett. 2000, 41, 6993.

4. Fujimoto, A.; Sakurai, A.; Synthesis 1977, 871.

5. Roudier, J. F.; Foucaud, A.; Synthesis 1984, 159.

6. Konkoy, C. S.; Fick, D. B.; Cai, S. X.; Lan, N. C.; Keana, J. F. W.; PCT Int Appl WO 0075123 2000; Chem. Abstr. 2001, 134, 29313a.

7. Burgard, A.; Lang, H. J.; Gerlach, U.; Tetrahedron 1999, 55, 7555.

8. Evans, J. M.; Fake, C. S.; Hamilton, T. C.; Poyser, R. H.; Showell, G. A.; J. Med. Chem. 1984, 27, 1127.

9. Evans, J. M.; Fake, C. S.; Hamilton, T. C.; Poyser, R. H.; Watts, E. A.; J. Med. Chem. 1983, 26, 1582.

10. Arnesto, D.; Horspool, W. M.; Martin, N.; Romos, A.; Seoane, C.; J. Org. Chem. 1989, 54, 3069.

11. Shi, D. Q.; Wang, X. S.; Tu, S. J.; Yao, C. S.; Wang, Y. C.; Jiegou Ниахие 2002, 21, 60.

12. Curini, M.; Epifano, F.; Chimichi, S.; Montanari, F.; Nochetti, M.; Rosati, O.; Tetrahedron Lett. 2005, 46, 3497.

13. Yadav, J. S.; Reddy, B. V. S.; Gupta, M. K.; Prathap, I.; Pandey, S. K.; Catal. Commun. 2007, 8, 2208.

14. Skaker, R. M.; Pharmazie 1996, 51, 148.

15. Abdolmohammadi, S.; Balalaie, S.; Tetrahedron Lett. 2007, 48, 3299.

16. Heravi, M. M.; Jani, B. A.; Derikvand, F.; Bamoharram, F. F.; Oskooie, H. A.; Catal. Commun. 2008, 10, 272.

17. Khurana, J. M.; Nand, B.; Saluja, P.; Tetrahedron 2010, 66, 5637. 
18. Kidwai, M.; Saxena, S.; Synth. Commun. 2006, 63, 2737.

19. Ghorbani-Vaghei, R.; Jalili, H.; Synthesis 2005, 1099.

20. Ghorbani-Vaghei, R.; Akbari-Dadamahaleh, S.; Tetrahedron Lett. 2009, 50, 1055.

21. Ghorbani-Vaghei, R.; Khazaei, A.; Tetrahedron Lett. 2003, 44, 7525.
22. Ghorbani-Vaghei, R.; Zolfigol, M.,A.; Chegeny, M.; Veisi, H.; Tetrahedron Lett. 2006, 47, 4505.

Submitted: September 20, 2010 Published online: February 3, 2011 


\title{
One-Pot Synthesis of $4 \mathrm{H}$-Chromene and Dihydropyrano[3,2-c]chromene Derivatives in Hydroalcoholic Media
}

\author{
Ramin Ghorbani-Vaghei, * Zahra Toghraei-Semiromi and Rahman Karimi-Nami
}

Department of Organic Chemistry, Faculty of Chemistry, Bu-Ali Sina University, 65174 Hamedan, Iran

Analytical data for selected compounds

2-Amino-3-cyano-4-(1,1-dicyanomethyl)-4H-chromene (Table 1, entry 1)

mp 147-148 ${ }^{\circ} \mathrm{C}$; IR (KBr) $v_{\max } / \mathrm{cm}^{-1}: 3486,3386$, 3181, 2201, 1643, 1600, 1568, 1408, 1236, 754, ${ }^{1} \mathrm{H}$ NMR (DMSO, $90 \mathrm{MHz}), \delta 4.82(\mathrm{~d}, 1 \mathrm{H}, J 3.5 \mathrm{~Hz}, \mathrm{CH}), 4.95$ (d, 1H, J $3.60 \mathrm{~Hz}, \mathrm{CH}), 6.69$ (s, 2H, NH$\left.{ }_{2}\right), 7.38$ (m, 4H, aromatic).

2-Amino-8-methoxy-3-cyano-4-(1,1-dicyanomethyl)-4Hchromene (Table 1, entry 2)

mp 188-189 ${ }^{\circ} \mathrm{C}$; IR (KBr) $v_{\max } / \mathrm{cm}^{-1}: 3464,3356,2910$, 2192, 1642, 1613, 1552, 1488, 1420, 1218, 750, ${ }^{1} \mathrm{H}$ NMR (DMSO, $90 \mathrm{MHz}), \delta 3.83\left(\mathrm{~s}, 3 \mathrm{H}, \mathrm{CH}_{3}\right), 4.53$ (d, 1H, J $3.8 \mathrm{~Hz}, \mathrm{CH}), 5.01$ (d, 1H, J $3.90 \mathrm{~Hz}, \mathrm{CH}), 7.07$ (m, 3H, aromatic), 7.52 (s, $2 \mathrm{H}, \mathrm{NH}_{2}$ ).

2-Amino-6-methoxy-3-cyano-4-(1,1-dicyanomethyl)-4Hchromene (Table 1, entry 3)

mp 184-185 ${ }^{\circ} \mathrm{C}$; IR (KBr) $v_{\max } / \mathrm{cm}^{-1}: 3402,3342$, 3218, 2185, 1659, 1587, 1426, 1217, 1037, 820, ${ }^{1} \mathrm{H}$ NMR (DMSO, $90 \mathrm{MHz}), \delta 3.73$ (s, 3H, $\mathrm{CH}_{3}$ ), 4.54 (s, 1H, CH), 5.09 (s, 1H, CH), 7.02 (s, 3H, aromatic), 7.41 (s, 2H, $\mathrm{NH}_{2}$ ). ${ }^{13} \mathrm{C}$ NMR [DMSO- $\left.d_{6}, 300 \mathrm{MHz}\right]: \delta 164.1,156.3,144.0$, 120.0, 119.1, 118.4, 117.7, 116.3, 113.6, 113.4, 113.4, 56.0, 48.79, 38.0. Anal. Calc. C, 63.15; H, 3.79; N, $21.04 \%$. Found: C, 62.90; H, 3.51; N, 20.65\%. $\mathrm{m} / \mathrm{z} 266$.

2-Amino-6-nitro-3-cyano-4-(1,1-dicyanomethyl)-4Hchromene (Table 1, entry 4)

mp 180-181 ${ }^{\circ} \mathrm{C}$; IR (KBr) $v_{\max } / \mathrm{cm}^{-1}: 3408,3319$, 3074, 2199, 1659, 1608, 1526, 1352, 1262, 748, ${ }^{1} \mathrm{H}$ NMR (DMSO, $90 \mathrm{MHz}$ ), $\delta 4.76$ (d, 2H, J $3.7 \mathrm{~Hz}, \mathrm{CH}$ ), 5.18 (d, $2 \mathrm{H}, J 3.7 \mathrm{~Hz}, \mathrm{CH}), 7.32-7.42(\mathrm{~m}, 1 \mathrm{H}$, aromatic), 7.77 (s, $2 \mathrm{H}, \mathrm{NH}_{2}$ ), 8.23-8.50 (m, 2H, aromatic).
2-Amino-6-bromo-3-cyano-4-(1,1-dicyanomethyl)-4Hchromene (Table 1, entry 5)

mp 163-164 ${ }^{\circ} \mathrm{C}$; IR (KBr) $v_{\max } / \mathrm{cm}^{-1}: 3471,3349$, 2885, 2189, 1635, 1597, 1596, 1426, 1228, 817, ${ }^{1} \mathrm{H}$ NMR (DMSO, $90 \mathrm{MHz}), \delta 4.76$ (d, 2H, J $3.5 \mathrm{~Hz}, \mathrm{CH}), 5.19$ (d, $2 \mathrm{H}, J 3.5 \mathrm{~Hz}, \mathrm{CH}), 7.72-7.96\left(\mathrm{~m}, 5 \mathrm{H}\right.$, aromatic and $\left.\mathrm{NH}_{2}\right)$.

2-Amino-6, 8-dibromo-3-cyano-4-(1,1-dicyanomethyl)-4Hchromene (Table 1, entry 6)

$\mathrm{mp} 182-183{ }^{\circ} \mathrm{C}$; IR (KBr) $v_{\max } / \mathrm{cm}^{-1}: 3414,3332$, 2885, 2197, 1655, 1600, 1562, 1457, 1433, 871, ' ${ }^{\mathrm{H}} \mathrm{NMR}$ (DMSO, $90 \mathrm{MHz}$ ), $\delta 4.76$ (d, 2H, J $3.6 \mathrm{~Hz}, \mathrm{CH}$ ), 5.17 (d, $2 \mathrm{H}, J 3.6 \mathrm{~Hz}, \mathrm{CH}), 7.74-7.96\left(\mathrm{~m}, 4 \mathrm{H}\right.$, aromatic and $\left.\mathrm{NH}_{2}\right)$.

2-Amino-6-chloro-3-cyano-4-(1,1-dicyanomethyl)-4Hchromene (Table 1, entry 7)

mp 151-154 ${ }^{\circ} \mathrm{C}$; IR (KBr) $v_{\max } / \mathrm{cm}^{-1}: 3445,3337$, 2862, 2194, 1644, 1600, 1572, 1457, 1483, 819, ${ }^{1} \mathrm{H}$ NMR (DMSO, $90 \mathrm{MHz}), \delta 4.62$ (d, 1H, J $3.8 \mathrm{~Hz}, \mathrm{CH}), 5.14$ (d, $1 \mathrm{H}, J 3.8 \mathrm{~Hz}, \mathrm{CH}), 7.10-7.57\left(\mathrm{~m}, 5 \mathrm{H}\right.$, aromatic and $\left.\mathrm{NH}_{2}\right)$, ${ }^{13} \mathrm{C}$ NMR [DMSO-d6, $300 \mathrm{MHz}$ ]: $\delta$ 163.7, 149.0, 130.5, 129.0, 128.9, 120.3, 119.6, 119.2, 118.8, 113.4, 113.2, 48.9, 37.3. Anal. Calc. C, 57.69; H, 2.61; N, $20.07 \%$. Found: C, 57.53; H, 2.36; N, 19.94\%. m/z 270.

2-Amino-5-oxo-4-phenyl-4,5-dihydropyrano[3,2-c] chromene-3-carbonitrile (Table 2, entry 1)

mp 260-264 ${ }^{\circ} \mathrm{C}$; IR (KBr) $v_{\max } / \mathrm{cm}^{-1}: 3379,3305,2196$, 1713, 1676, 1637, ${ }^{1} \mathrm{H}$ NMR (DMSO, $90 \mathrm{MHz}$ ), $\delta 4.45$ (s, $1 \mathrm{H}, \mathrm{CH}), 7.28-7.86\left(\mathrm{~m}, 11 \mathrm{H}\right.$, aromatic and $\left.\mathrm{NH}_{2}\right)$.

2-Amino-4-(4-chlorophenyl)-5-oxo-4,5-dihydropyrano[3,2-c]chromene-3-carbonitrile (Table 2, entry 2)

$\mathrm{mp} 265-267^{\circ} \mathrm{C}$; IR (KBr) $v_{\max } / \mathrm{cm}^{-1}: 3380,3291,3189$, 2191, 1713, 1676, ${ }^{1} \mathrm{H}$ NMR (DMSO, $90 \mathrm{MHz}$ ), $\delta 4.72$ (s, $1 \mathrm{H}, \mathrm{CH}), 7.40-8.13\left(\mathrm{~m}, 10 \mathrm{H}\right.$, aromatic and $\left.\mathrm{NH}_{2}\right)$. 
2-Amino-4-(4-methoxyphenyl)-5-oxo-4,5-dihydropyrano[3,2-c]chromene-3-carbonitrile (Table 2, entry 3)

$\mathrm{mp} 246-249^{\circ} \mathrm{C}$; IR (KBr) $v_{\max } / \mathrm{cm}^{-1}: 3500,3450,3350$, 2208, 1647,1174, ${ }^{1} \mathrm{H}$ NMR (DMSO, $90 \mathrm{MHz}$ ), $\delta 3.71$ (s, $\left.3 \mathrm{H}, \mathrm{CH}_{3}\right), 4.39(\mathrm{~s}, 1 \mathrm{H}, \mathrm{CH}), 6.81-7.85(\mathrm{~m}, 10 \mathrm{H}$, aromatic and $\mathrm{NH}_{2}$ ).

2-Amino-5-oxo-4-p-tolyl-4,5-dihydropyrano[3,2-c] chromene-3-carbonitrile (Table 2, entry 4)

$\mathrm{mp} 252-254^{\circ} \mathrm{C}$; IR (KBr) $v_{\max } / \mathrm{cm}^{-1}: 3388,3311,3189$, 2194, 1713, 1676, 1637, ${ }^{1} \mathrm{H}$ NMR (DMSO, $90 \mathrm{MHz}$ ), $\delta 2.25\left(\mathrm{~s}, 3 \mathrm{H}, \mathrm{CH}_{3}\right), 4.40(\mathrm{~s}, 1 \mathrm{H}, \mathrm{CH}), 7.12-7.93(\mathrm{~m}, 10 \mathrm{H}$, aromatic and $\left.\mathrm{NH}_{2}\right)$.

2-Amino-4-(4-nitrophenyl)-5-oxo-4,5-dihydropyrano[3,2-c]chromene-3-carbonitrile (Table 2, entry 5)

mp 255-258 ${ }^{\circ} \mathrm{C}$; IR (KBr) $v_{\max } / \mathrm{cm}^{-1}: 3482,3370,3071$, 2196, 1718, 1673, 1606, 1505,1373, 1348, 766. ${ }^{1} \mathrm{H}$ NMR (DMSO, $90 \mathrm{MHz}$ ), $\delta 4.66$ (s, 1H, CH), 7.47-8.21 (m, 10H, aromatic and $\mathrm{NH}_{2}$ ).

2-Amino-4-(3-nitrophenyl)-5-oxo-4,5-dihydropyrano[3,2-c]chromene-3-carbonitrile (Table 2, entry 6)

mp 256-259 ${ }^{\circ} \mathrm{C}$; IR (KBr) $v_{\max } / \mathrm{cm}^{-1}: 3408,3324,3192$, 2194, 1710, 1677, 1608, 1528,1380, 1347, 764. ${ }^{1} \mathrm{H}$ NMR (DMSO, $90 \mathrm{MHz}$ ), $\delta 4.72$ (s, 1H, CH), 7.54-8.12 (m, 10H, aromatic and $\mathrm{NH}_{2}$ ).

2-Amino-4-(4-bromophenyl)-5-oxo-4,5-dihydropyrano[3,2-c]chromene-3-carbonitrile (Table 2, entry 7)

mp 255-257 ${ }^{\circ} \mathrm{C}$; IR (KBr) $v_{\max } / \mathrm{cm}^{-1}: 3387,3309,3188$, 2181, 1710, 1677, 1602, 1577,1062, 758. ${ }^{1} \mathrm{H}$ NMR (DMSO, $90 \mathrm{MHz}), \delta 4.46(\mathrm{~s}, 1 \mathrm{H}, \mathrm{CH}), 7.19-7.84(\mathrm{~m}, 10 \mathrm{H}$, aromatic and $\mathrm{NH}_{2}$ ).
2-Amino-4-(2,4-dichlorophenyl)-5-oxo-4,5-dihydropyrano[3,2-c]chromene-3-carbonitrile (Table 2, entry 8)

$\mathrm{mp} 255-258^{\circ} \mathrm{C}$; IR (KBr) $v_{\max } / \mathrm{cm}^{-1}: 3459,3290,3163$, 2199, 1716, 1673, 1630, 1589,1061, 761. ' $\mathrm{H}$ NMR (DMSO, $90 \mathrm{MHz}), \delta 4.94(\mathrm{~s}, 1 \mathrm{H}, \mathrm{CH}), 7.34-7.92(\mathrm{~m}, 9 \mathrm{H}$, aromatic and $\mathrm{NH}_{2}$ ).

2-Amino-4-(2,3-dichlorophenyl)-5-oxo-4,5-dihydropyrano[3,2-c]chromene-3-carbonitrile (Table 2, entry 9)

mp 273-276 ${ }^{\circ} \mathrm{C}$; IR (KBr) $v_{\max } / \mathrm{cm}^{-1}: 3405,3305,3181$, 2199, 1711, 1674, 1602, 1492,1062, 764. ${ }^{1} \mathrm{H}$ NMR (DMSO, $90 \mathrm{MHz}), \delta 5.07(\mathrm{~s}, 1 \mathrm{H}, \mathrm{CH}), 7.33-7.96(\mathrm{~m}, 9 \mathrm{H}$, aromatic and $\mathrm{NH}_{2}$ ).

2-Amino-4-(2,6-dichlorophenyl)-5-oxo-4,5-dihydropyrano[3,2-c]chromene-3-carbonitrile (Table 2, entry 10) mp 274-277 ${ }^{\circ} \mathrm{C}$; IR (KBr) $v_{\max } / \mathrm{cm}^{-1}: 3419,3277,3173$, 2200, 1707, 1673, 1633, 1599,1379, 758. ${ }^{1} \mathrm{H}$ NMR (DMSO, $90 \mathrm{MHz}), \delta 5.51(\mathrm{~s}, 1 \mathrm{H}, \mathrm{CH}), 7.35-7.92(\mathrm{~m}, 9 \mathrm{H}$, aromatic and $\mathrm{NH}_{2}$ ).

2-Amino-5-oxo-4-(3,4,5-trimethoxyphenyl)-4,5-dihydropyrano[3,2-c]chromene-3-carbonitrile (Table 2, entry 11)

mp 224-226 ${ }^{\circ} \mathrm{C}$; IR (KBr) $v_{\max } / \mathrm{cm}^{-1}: 3425,3321,2191$, $1672,1595,1375,1154,{ }^{1} \mathrm{H}$ NMR (DMSO, $\left.90 \mathrm{MHz}\right), \delta 3.63$ (s, $\left.3 \mathrm{H}, \mathrm{CH}_{3}\right), 3.71\left(\mathrm{~s}, 6 \mathrm{H}, \mathrm{CH}_{3}\right), 4.43(\mathrm{~s}, 1 \mathrm{H}, \mathrm{H}), 6.52(\mathrm{~s}, 2 \mathrm{H}$, $\mathrm{NH}_{2}$ ), 7.36-7.93 (m, 6H, aromatic), ${ }^{13} \mathrm{C}$ NMR [DMSO-d6, $300 \mathrm{MHz}]: \delta 160.1,158.5,154.0,153.3,152.6,139.4$, 137.1, 133.3, 125.1, 123.0, 119.7, 117.0, 113.6, 105.4, 104.1, 60.4, 58., 56., 37.7. Anal. Calc. C, 65.02; H, 4.46; N, 6.89\%. Found: C, 65.0; H, 4.27; N, 6.93\%. m/z 404.

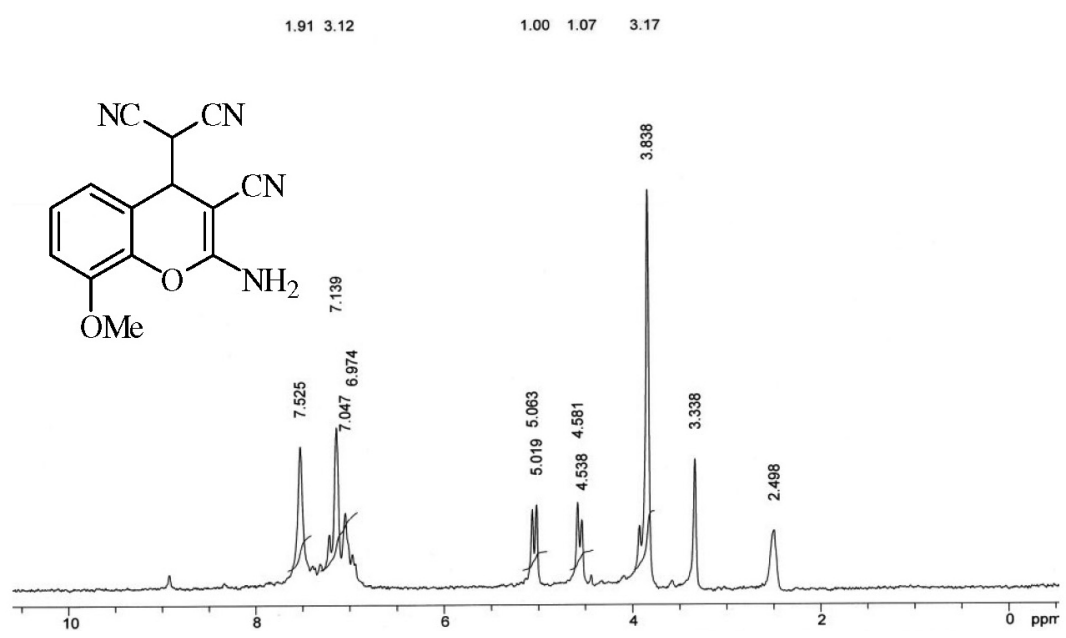

Figure S1. 'H NMR (DMSO) 2-amino-8-methoxy-3-cyano-4-(1,1-dicyanomethyl)-4H-chromene (Table 1, entry 2). 


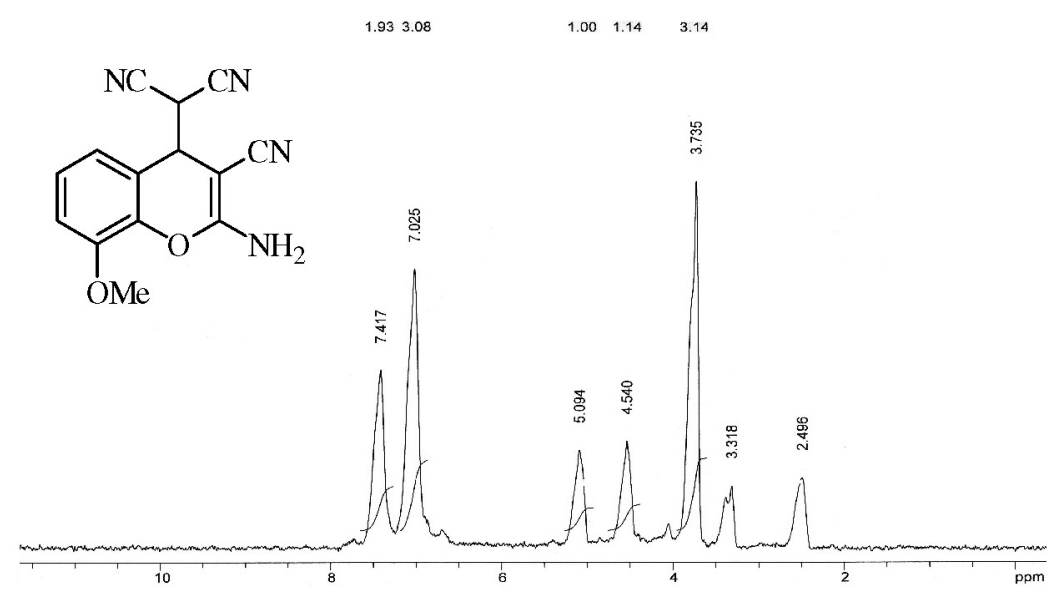

Figure S2. 'H NMR (DMSO) 2-amino-6-methoxy-3-cyano-4-(1,1-dicyanomethyl)-4H-chromene (Table 1, entry 3).

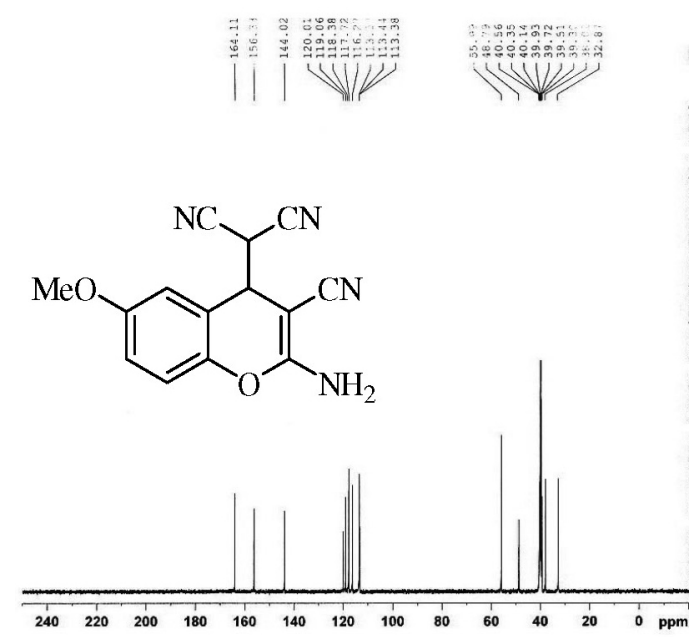

Figure S3. ${ }^{13} \mathrm{C}$ NMR (DMSO) 2-amino-6-methoxy-3-cyano-4-(1,1-dicyanomethyl)-4H-chromene (Table 1, entry 3).

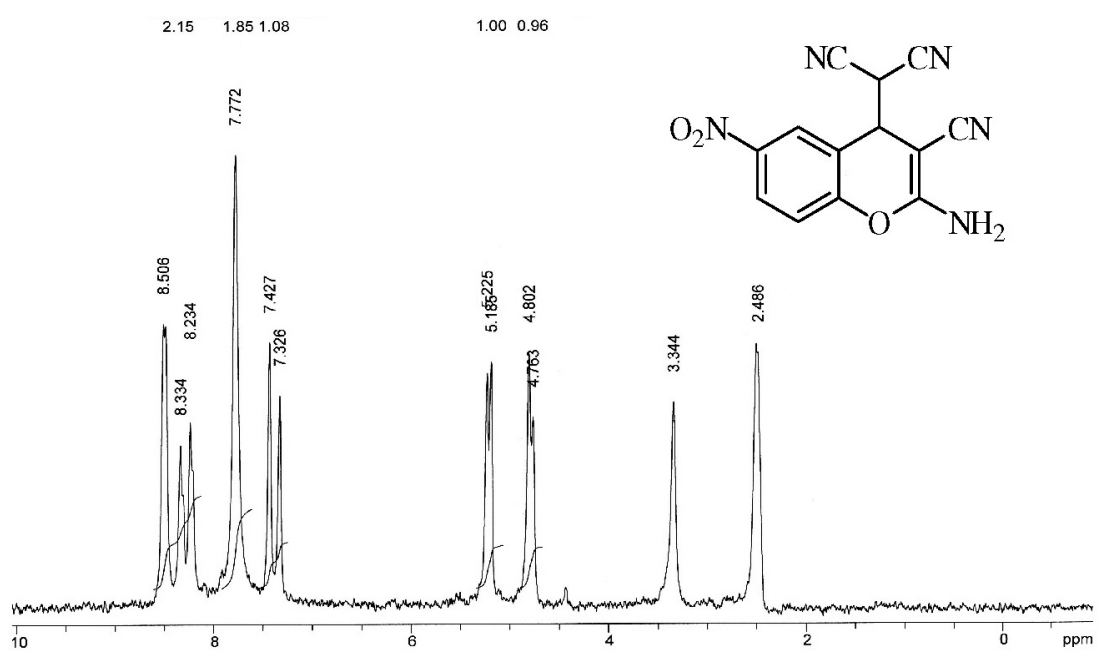

Figure S4. ${ }^{1} \mathrm{H}$ NMR (DMSO) 2-amino-6-nitro-3-cyano-4-(1,1-dicyanomethyl)-4H-chromene (Table 1, entry 4). 


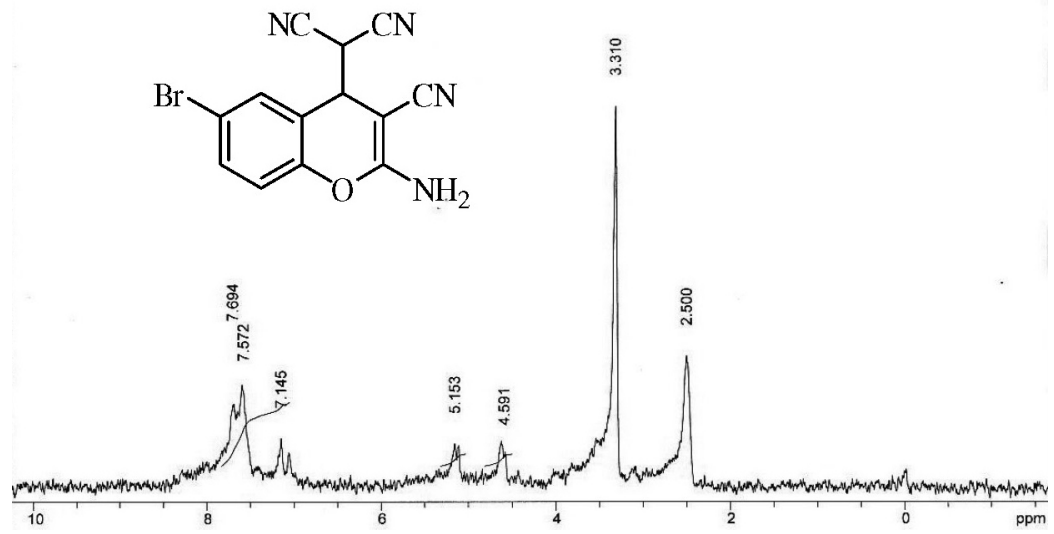

$\underset{\substack{0 \\ j}}{ }$

Figure S5. 'H NMR (DMSO) 2-amino-6-bromo-3-cyano-4-(1,1-dicyanomethyl)-4H-chromene (Table 1, entry 5).

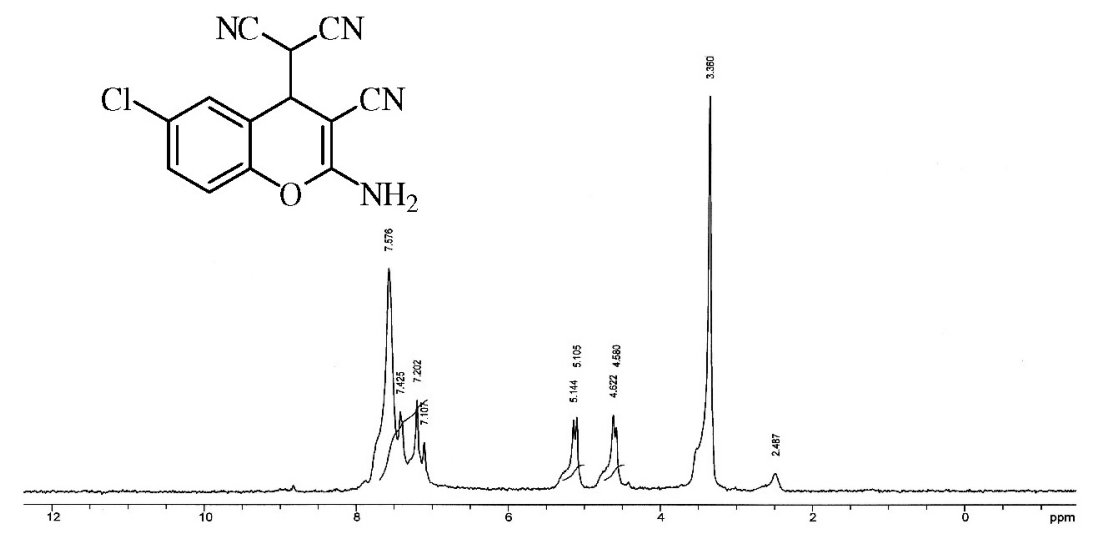

Figure S6. ${ }^{1} \mathrm{H}$ NMR (DMSO) 2-amino-6-chloro-3-cyano-4-(1,1-dicyanomethyl)-4H-chromene (Table 1, entry 7).

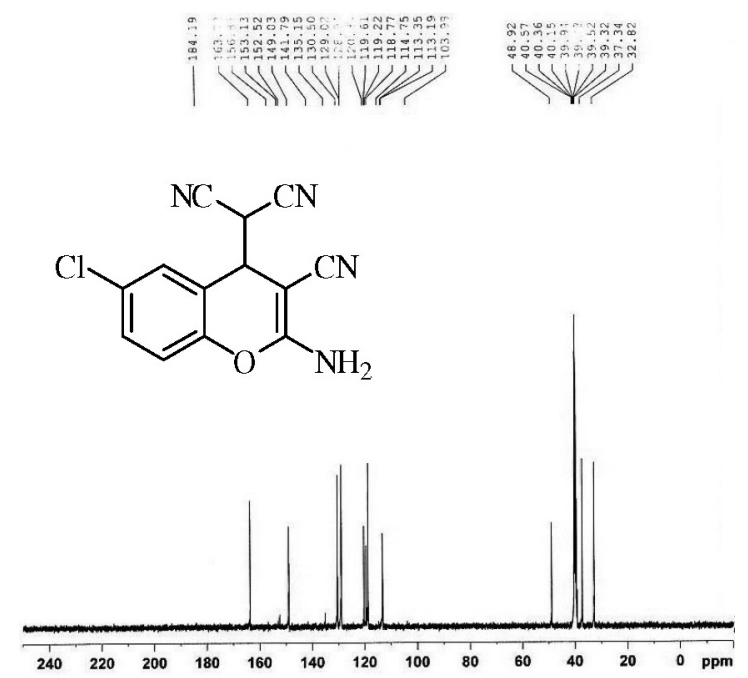

Figure S7. ${ }^{13} \mathrm{C}$ NMR (DMSO) 2-amino-6-chloro-3-cyano-4-(1,1-dicyanomethyl)-4H-chromene (Table 1, entry 7). 


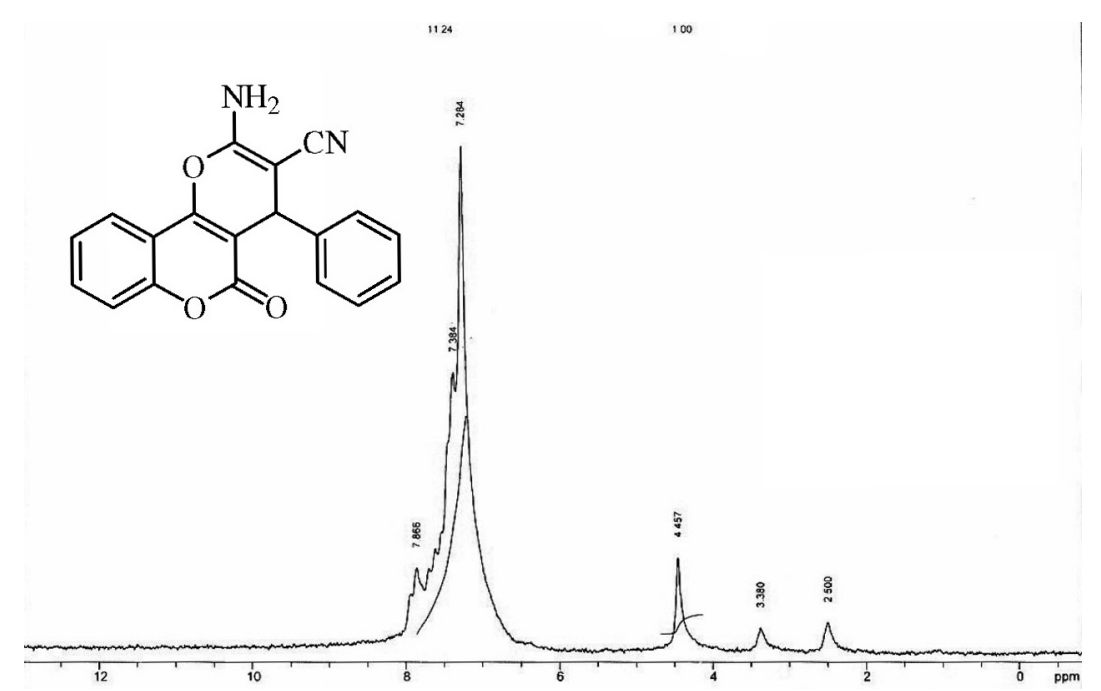

Figure S8. ${ }^{1} \mathrm{H}$ NMR (DMSO) 2-amino-5-oxo-4-phenyl-4,5-dihydropyrano[3,2-c]chromene-3-carbonitrile (Table 2, entry 1).

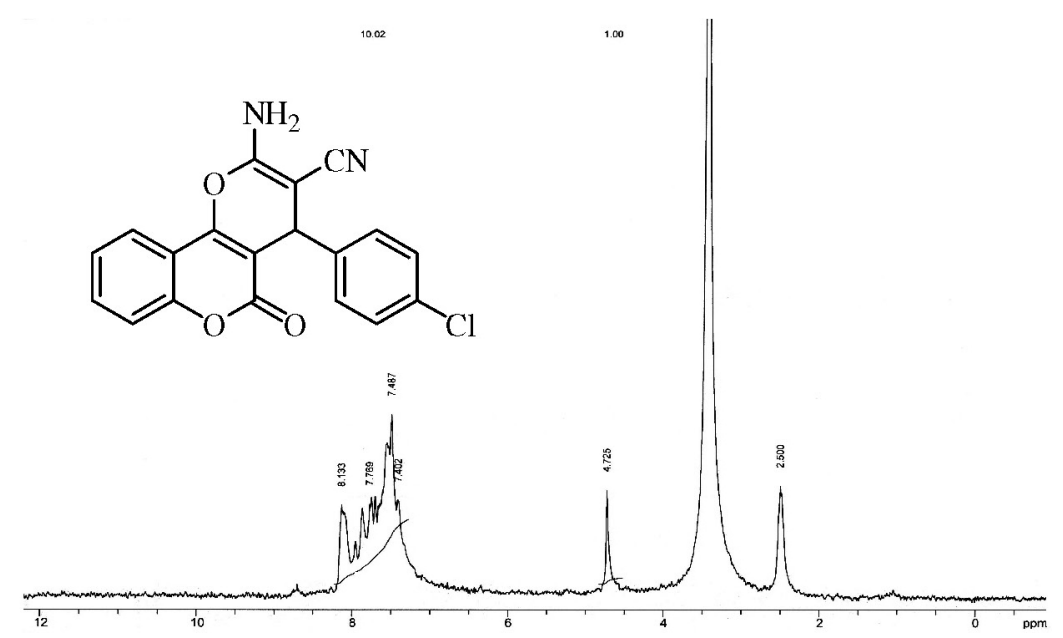

Figure S9. 'H NMR (DMSO) 2-amino-4-(4-chlorophenyl)-5-oxo-4,5-dihydropyrano[3,2-c]chromene-3-carbonitrile (Table 2, entry 2).

$8.79 \quad 2.27$<smiles>COc1ccc(C2C(C#N)=C(N)Oc3c2c(=O)oc2ccccc32)cc1</smiles>

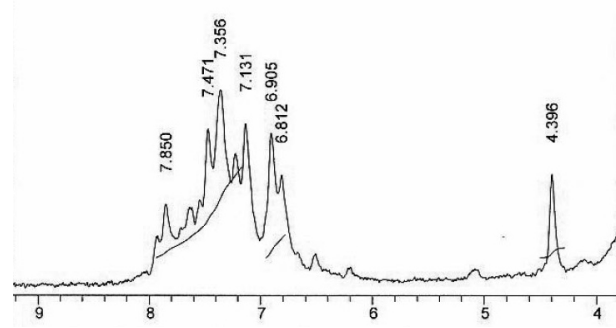

곯

$\mid$ 3.07

$¥$ 兽
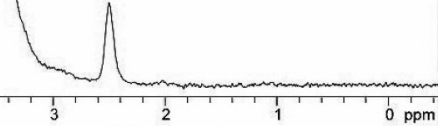

Figure S10. ${ }^{1}$ H NMR (DMSO) 2-amino-4-(4-methoxyphenyl)-5-oxo-4,5-dihydropyrano[3,2-c]chromene-3-carbonitrile (Table 2, entry 3). 


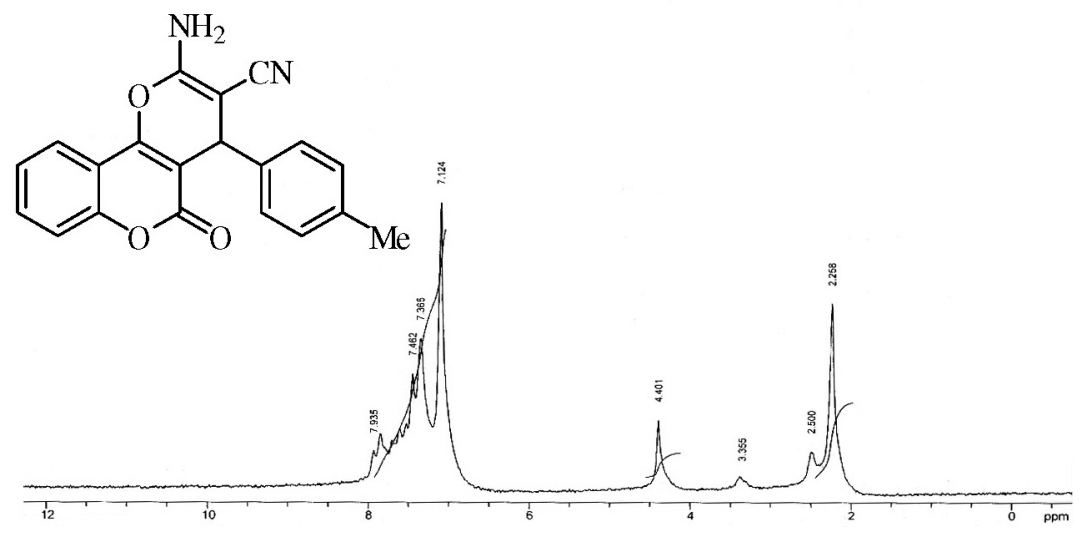

Figure S11. ${ }^{1} \mathrm{H}$ NMR (DMSO) 2-amino-5-oxo-4-p-tolyl-4,5-dihydropyrano[3,2-c]chromene-3-carbonitrile (Table 2, entry 4).

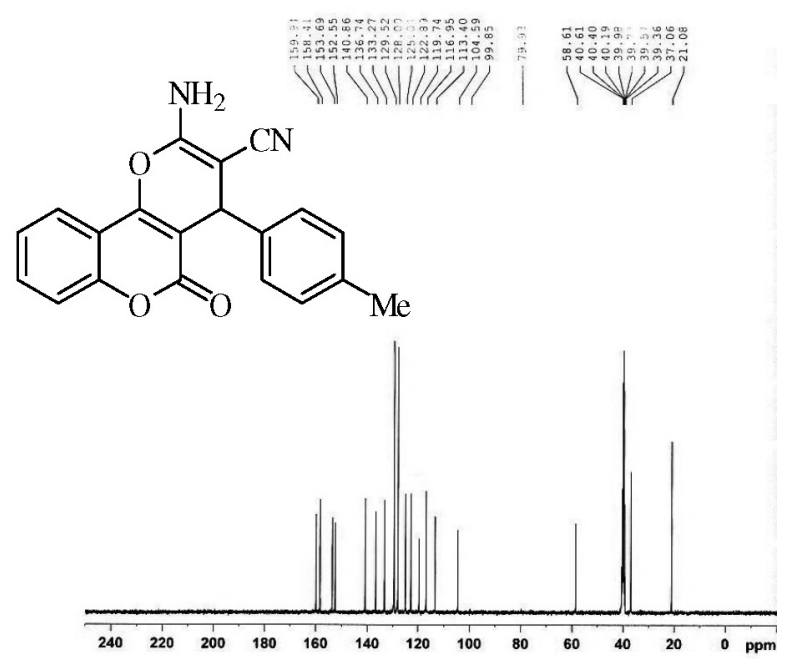

Figure S12. ${ }^{13} \mathrm{C}$ NMR (DMSO) 2-amino-5-oxo-4-p-tolyl-4,5-dihydropyrano[3,2-c]chromene-3-carbonitrile (Table 2, entry 4).

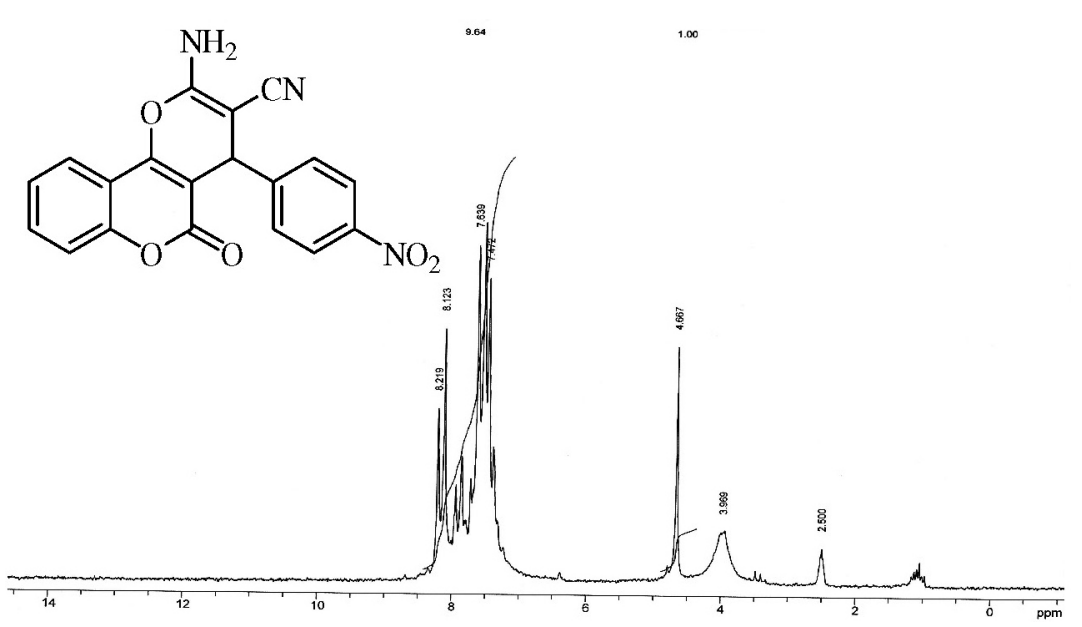

Figure S13. ${ }^{~} \mathrm{H}$ NMR (DMSO) 2-amino-4-(4-nitrophenyl)-5-oxo-4,5-dihydropyrano[3,2-c]chromene-3-carbonitrile (Table 2, entry 5). 


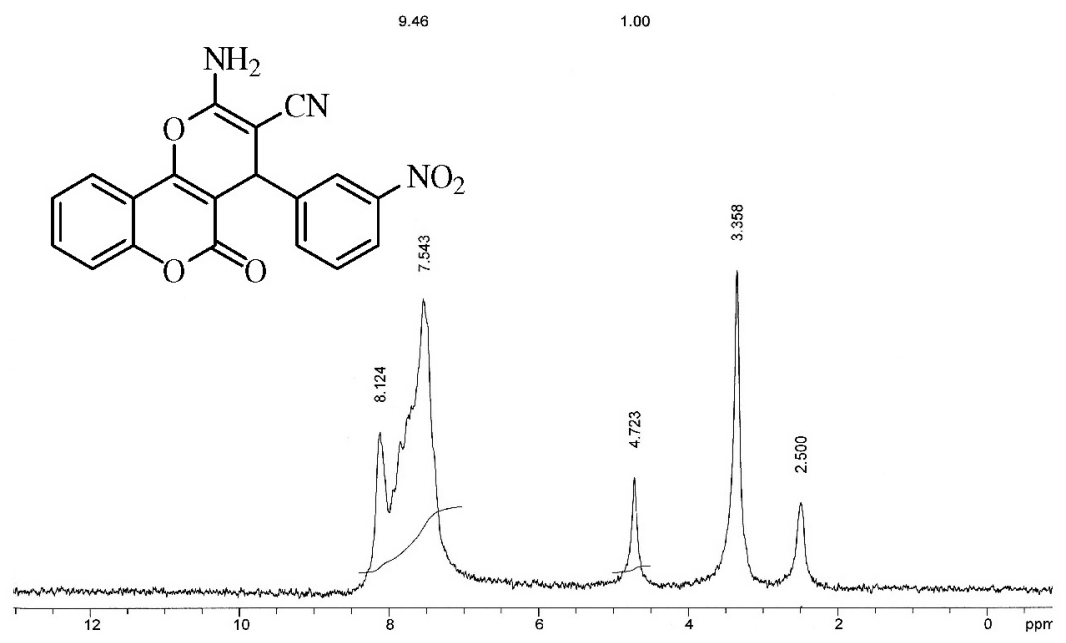

Figure S14. 'H NMR (DMSO) 2-amino-4-(3-nitrophenyl)-5-oxo-4,5-dihydropyrano[3,2-c]chromene-3-carbonitrile (Table 2, entry 6).

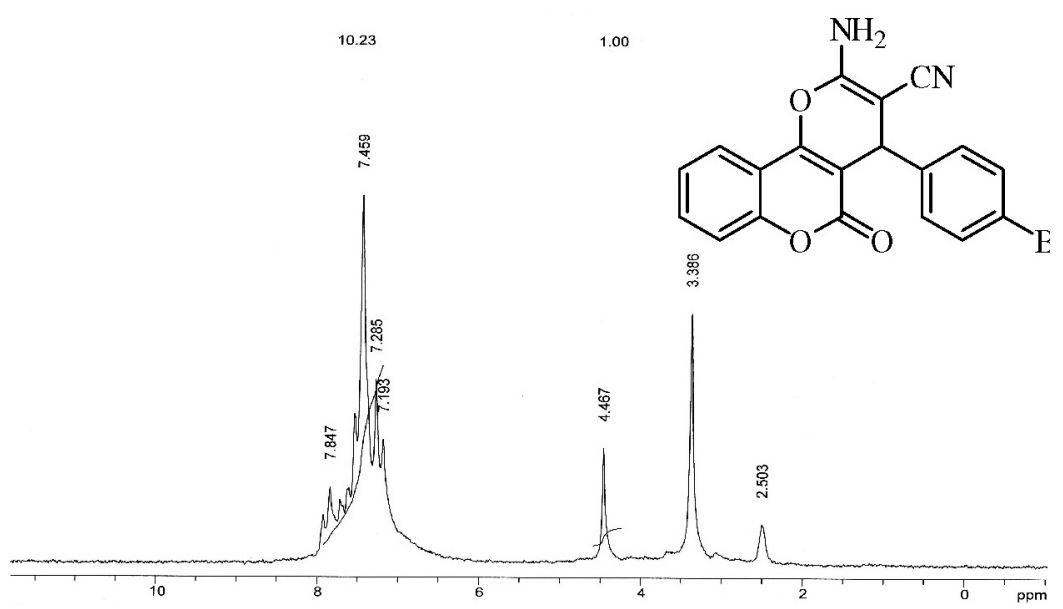

Figure S15. 'H NMR (DMSO) 2-amino-4-(4-bromophenyl)-5-oxo-4,5-dihydropyrano[3,2-c]chromene-3-carbonitrile (Table 2, entry 7).

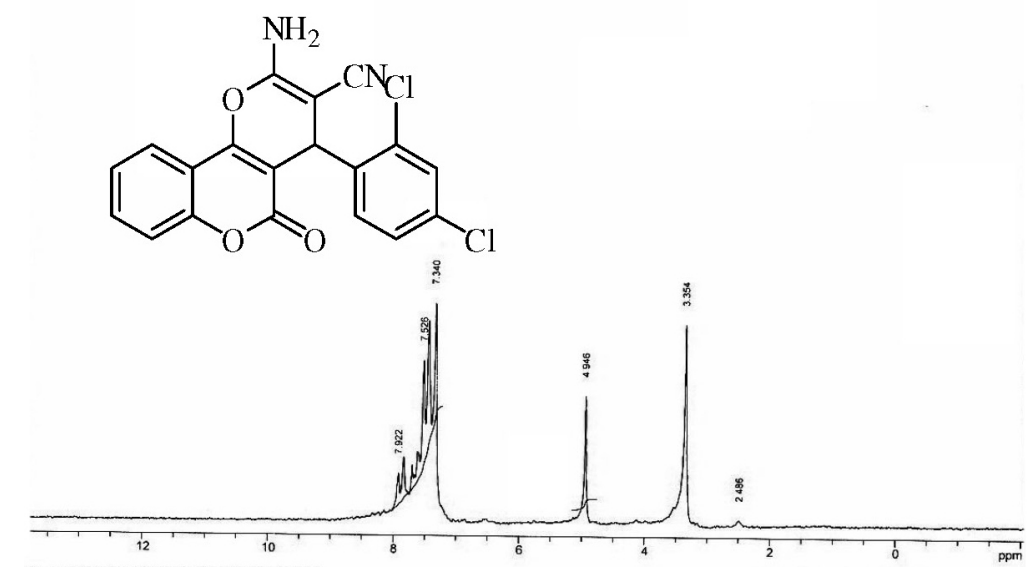

Figure S16. ${ }^{1} \mathrm{H}$ NMR (DMSO) 2-amino-4-(2,4-dichlorophenyl)-5-oxo-4,5-dihydropyrano[3,2-c]chromene-3-carbonitrile (Table 2, entry 8). 


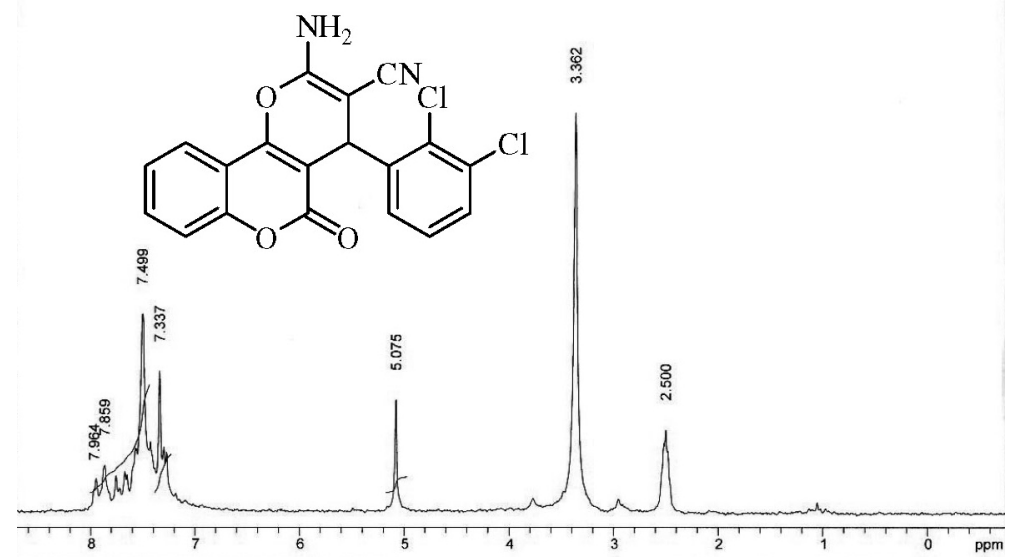

Figure S17. ${ }^{1} \mathrm{H}$ NMR (DMSO) 2-amino-4-(2,3-dichlorophenyl)-5-oxo-4,5-dihydropyrano[3,2-c]chromene-3-carbonitrile (Table 2, entry 9).

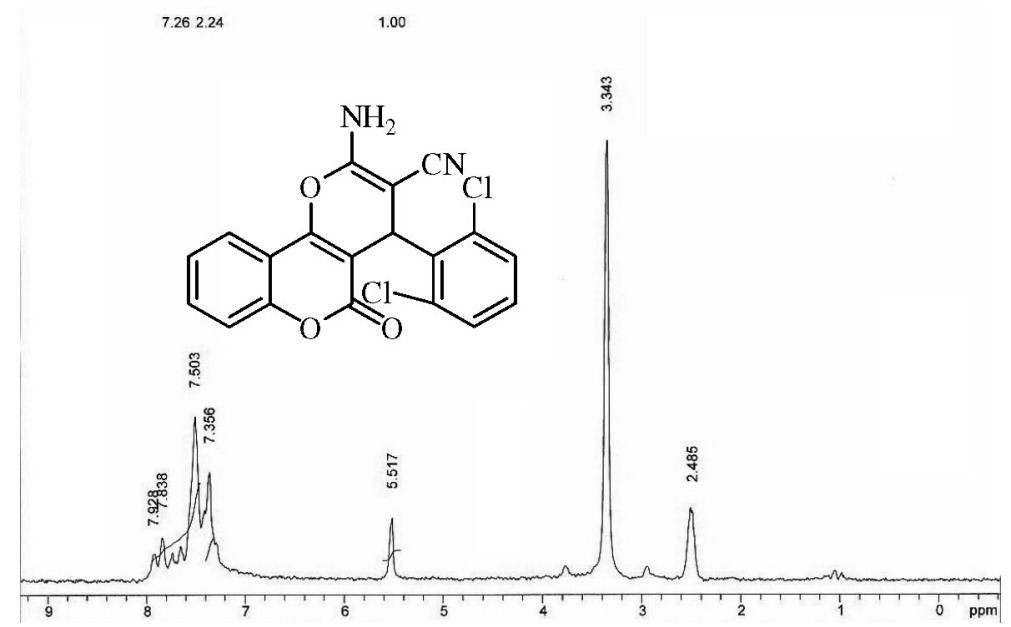

Figure S18. 'H NMR (DMSO) 2-amino-4-(2,6-dichlorophenyl)-5-oxo-4,5-dihydropyrano[3,2-c]chromene-3-carbonitrile (Table 2, entry 10).

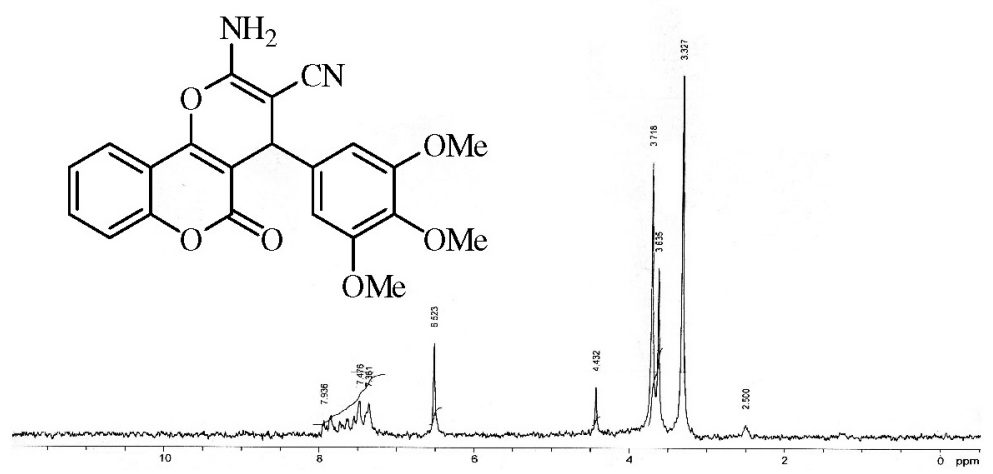

Figure S19. ${ }^{1} \mathrm{H}$ NMR (DMSO) 2-amino-5-oxo-4-(3,4,5-trimethoxyphenyl)-4,5-dihydropyrano[3,2-c]chromene-3-carbonitrile (Table 2, entry 11). 

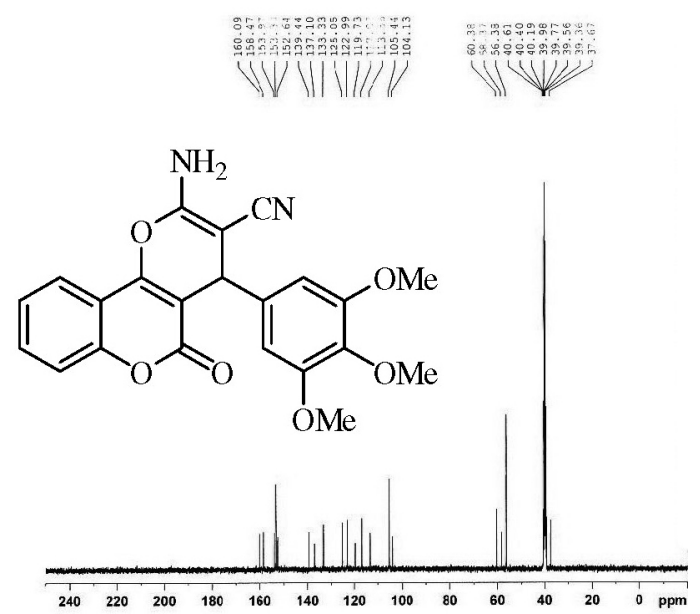

Figure S20. ${ }^{13} \mathrm{C}$ NMR (DMSO) 2-amino-5-oxo-4-(3,4,5-trimethoxyphenyl)-4,5-dihydropyrano[3,2-c]chromene-3-carbonitrile (Table 2, entry 11). 